\title{
LnCRNA RNCR3 promotes Chop expression by sponging miR- 185-5p during MDSC differentiation
}

\author{
Wencong Shang ${ }^{1,2,3}$, Zhenzhen Tang ${ }^{1,2,3}$, Yunhuan Gao ${ }^{1,2,3}$, Houbao Qi ${ }^{1,2,3}$, Xiaomin \\ Su ${ }^{1,2,3}$, Yuan Zhang ${ }^{1,2,3}$ and Rongcun Yang ${ }^{1,2,3}$ \\ ${ }^{1}$ State Key Laboratory of Medicinal Chemical Biology, Nankai University, Tianjin, China \\ ${ }^{2}$ Key Laboratory of Bioactive Materials, Ministry of Education, Nankai University, Tianjin, China \\ ${ }^{3}$ Department of Immunology, Nankai University School of Medicine, Nankai University, Tianjin, China \\ Correspondence to: Rongcun Yang, email: ryang@nankai.edu.cn \\ Keywords: myeloid-derived suppressor cells; RNCR3; miR-185-5p; Chop; epigenetic modification \\ Received: July 13,2017 Accepted: November 17, $2017 \quad$ Published: December 04, 2017 \\ Copyright: Shang et al. This is an open-access article distributed under the terms of the Creative Commons Attribution License 3.0 \\ (CC BY 3.0), which permits unrestricted use, distribution, and reproduction in any medium, provided the original author and source \\ are credited.
}

\section{ABSTRACT}

Myeloid-derived suppressor cells (MDSCs) play a critical role in regulating immune responses in cancer and other pathological conditions. Mechanism(s) regulating MDSC differentiation and function is not completely clear, especially epigenetic regulation. In this study, we found that MDSCs express retinal non-coding RNA3 (RNCR3), and the expression in MDSCs is upregulated by inflammatory and tumor associated factors. RNCR3 may function as a competing endogenous RNA (ceRNA) to promote Chop expression by sponging miR-185-5p during MDSC differentiation. RNCR3 knockdown suppressed differentiation and function of MDSCs in vitro and in vivo. Quantitative RT-PCR showed that RNCR3 was negatively regulated by miR-185-5p in MDSCs. MiR185-5p affected the expansion of MDSCs and reversed the effect of RNCR3 on MDSC differentiation and function through directly targeting Chop. Thus, our results suggest a RNCR3/miR-185-5p/Chop autologously strengthening network to promote MDSC differentiation and suppressive function in response to extracellular inflammatory and tumor-associated signals.

\section{INTRODUCTION}

Myeloid-derived suppressor cells (MDSCs) have emerged as major regulators of immune responses in cancer and other pathological conditions [1-3]. In mice, MDSCs are identified by expressing both CD11b and Gr1 markers, and divided into two major subsets including polymorphonuclear MDSC (PMN-MDSC) defined as CD $11 b^{+}{ }^{+}$y6G $\mathrm{G}^{+}$Ly6 $\mathrm{C}^{\text {lo }}$ and monocytic MDSC (M-MDSC) defined as CD11b+Ly6G-Ly6Chi $[4,5]$. These cells express high levels of arginase-1(Arg-1), nitric oxide synthase 2 (NOS2/iNOS), NADPH oxidase 2 (NOX2) and prostaglandin endoperoxide synthase 2 (Ptgs2, also called $\mathrm{COX} 2$ ), resulting in production of nitric oxide (NO) and reactive oxygen species (ROS) [6,7]. NO is labile and reacts with multiple compounds to produce many toxic and regulatory factors. ROS including hydrogen peroxide
$\left(\mathrm{H}_{2} \mathrm{O}_{2}\right)$, hydroxyl radical, and hypochlorous acid can damage proteins, lipids, and nucleic acids [8]. Whereas a high level of Arg-1 may cause elimination of key nutrition factors needed for $\mathrm{T}$ cell proliferation by depleting local environment L-arginine [9], L-cysteine [10] or tryptophan levels [11].

MDSCs are generated by sustained myelopoiesis during chronic inflammation, autoimmune diseases or cancer $[1,4,12]$. These MDSCs may also be induced in vitro by different cytokines including G-CSF, IL-6, GMCSF, IL-1 $\beta$, PEG2, VEGF and TNF $\alpha$ [13-17]. Notably, there exists remarkable difference in the constitution of subsets and suppressive function of MDSCs in response to different cytokines such as that GM-CSF plus IL-6 induced MDSCs have higher inhibitory activity than other MDSCs [18]. Multiple transcription factors and signaling pathways such as STAT3 (signal transducer of activator 
of transcription 3), C/EBP $\beta$ (CCAAT/enhancer binding protein $\beta$ ) and Chop (C/EBP homologous protein; encoded by Ddit3 and also known as Chop-10 and Gadd153) [19, 20] are involved in the regulation of MDSC differentiation and function $[4,13,14]$. However, differentiation and function of immune cells may be also epigenetically or post-translationally regulated. Emerging evidences show that long non-coding RNAs (lncRNAs) play critical roles in both developmental and differentiation processes by controlling gene expression. LncRNAs, that are longer than 200 nucleotides, are defined as a class of transcripts with no protein-coding potential. They can be either intergenic (between protein coding genes, long intergenic noncoding RNA (lincRNA)), intronic, natural antisense transcripts, or transcribed from divergent enhancers and promoters. So far, many lncRNAs have been shown to regulate the development and function of myeloid cells [21-23]. We have also demonstrated that lncRNA HOTAIRM1 can regulate peripheral blood cells to differentiate into dendritic cells (DCs) by sponging miR3960 to regulate HOXA1 expression [24]. However, the effect(s) of lncRNA on the differentiation and function of MDSCs is very little understood. The understanding of specific molecular mechanism(s) responsible for MDSC differentiation and function would enable more precise therapeutics by targeting these cells.

RNCR3 (retinal noncoding RNA3, known as LINC00599 in human), a lncRNA transcribed from the intergenic regions of the genome and conserved in mammals [25], can regulate proliferation and/or function of different kinds of cells including neurons, oligodendrocyte, EC (endothelial cell), VSMC (vascular smooth muscle cell) and retinal endothelial cell [25-28]. Interestingly, RNCR3 knockdown also results in higher levels of inflammatory factors such as TNF- $\alpha$, CCL2, and IL-6 in blood plasma, implying that RNCR3 may play a role in immune system [28]. In this study, we demonstrate that MDSCs express RNCR3, and this expression is significantly upregulated in inflammatory and tumor microenvironment. Importantly, we found that RNCR3 promotes MDSC differentiation and function by sponging miR-185-5p to release its target gene Chop. This may provide a novel regulatory mechanism for MDSC differentiation and suppressive function.

\section{RESULTS}

\section{Tumor microenvironment upregulates expression of RNCR3 in MDSCs}

LncRNA RNCR3 (2998 bp) is mainly expressed in brain (www.lncrnadb.org). However, RNCR3 is also detected in EC (endothelial cell), VSMC (vascular smooth muscle cell) and retinal endothelial cell [25-28]. We here found that MDSCs also express RNCR3. RNCR3 could be detected using RT-PCR and confirmed by sequencing analyses in MDSCs (Supplementary Figure 1). This IncRNA is upregulated in aortic atherosclerotic lesions with hypoxia, oxidative stress, or inflammatory stress [28], which are shared by tumor environment. Indeed, RNCR3 expression in MDSCs could be upregulated in tumor microenvironment. MDSCs from the spleens of B16 tumor-bearing mice had higher levels of RNCR3 than those from the spleens of tumor-free mice (Figure $1 \mathrm{~A}$ and 1B). The levels of RNCR3 were much higher in $\mathrm{CD} 11 \mathrm{~b}^{+} \mathrm{Gr} 1^{+} \mathrm{MDSC}$ s isolated from tumor tissues than splenic $\mathrm{CD} 11 \mathrm{~b}^{+} \mathrm{Gr} 1^{+} \mathrm{MDSC}$ s or bone marrow $\mathrm{CD} 11 \mathrm{~b}^{+} \mathrm{Gr} 1^{+} \mathrm{MDSC}$ (Figure 1B). To further explore factor(s) regulating RNCR3 expression in tumor microenvironment, we used different inflammatory factors to induce MDSCs from BMCs. We found that the levels of RNCR3 was much higher in $\mathrm{CD} 11 \mathrm{~b}^{+} \mathrm{Gr} 1^{+}$MDSCs after exposing to GM-CSF plus IL-6 as compared to those only exposed to GM-CSF (Figure 1C), indicating that IL-6 may be a critical factor in inducing RNCR3 expression. Since B16 melanoma cells do not produce IL-6 ([29] and not shown), IL-6 may be from tumor inflammatory environments not from B16 melanoma cells. Indeed, the levels of RNCR3 were markedly lower in the MDSCs isolated from bone marrow of IL-6 knockout (IL-6 ${ }^{-/}$) mice bearing B16 tumor than those in WT mice (Figure 1D). Taken together, these results indicate that tumor microenvironment factors such as IL-6 may promote the expression of RNCR3 during MDSC differentiation, implying that RNCR3 may play an important role in the differentiation and function of MDSCs.

\section{RNCR3 knockdown interrupts MDSC differentiation in vitro}

To investigate the effect(s) of RNCR3 on the differentiation and function of MDSCs, we employed two kinds of knockdown methods including RNCR3 siRNA and RNCR3 shRNA. BMCs were transfected or transduced with RNCR3 siRNA or RNCR3 shRNA/ lentivirus, and cultured in vitro for 4 days in the presence of GM-CSF and IL-6 to observe the effect of RNCR3 knockdown on the differentiation of MDSCs. We first designed two different RNCR3 siRNAs. Quantitative RT-PCR (qRT-PCR) revealed that the level of RNCR3 was reduced by RNCR3 siRNA transfection (Figure 2A). RNCR3 siRNA with stronger silencing efficiency was selected for subsequent assay. We found that RNCR3 knockdown decreased the percentage of $\mathrm{Gr}^{+}{ }^{+} \mathrm{CD} 11 \mathrm{~b}^{+}$ cells as compared to control (Figure 2B and 2C). Further analyses showed that the percentage of PMN-MDSCs was significantly reduced; whereas the proportion of M-MDSCs increased in these RNCR3 knockdown MDSCs (Figure 2B and 2C), indicating that RNCR3 is involved in the regulation of MDSC differentiation. We also prepared shRNA/lentiviruses with remarkably knockdown ability and over $90 \%$ transduction rate (Figure 
A

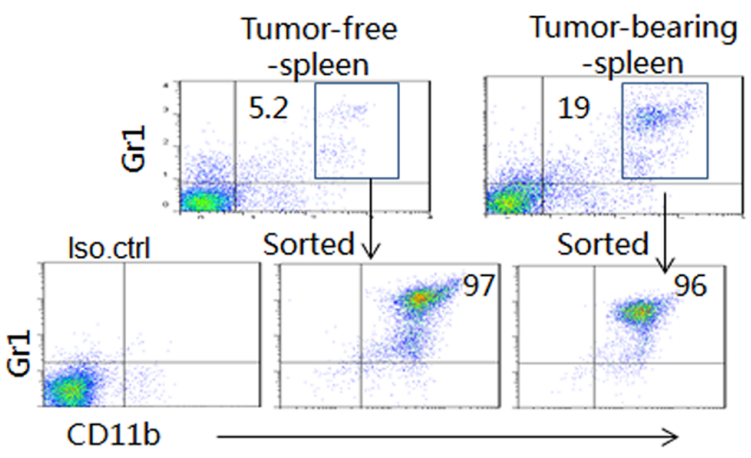

B

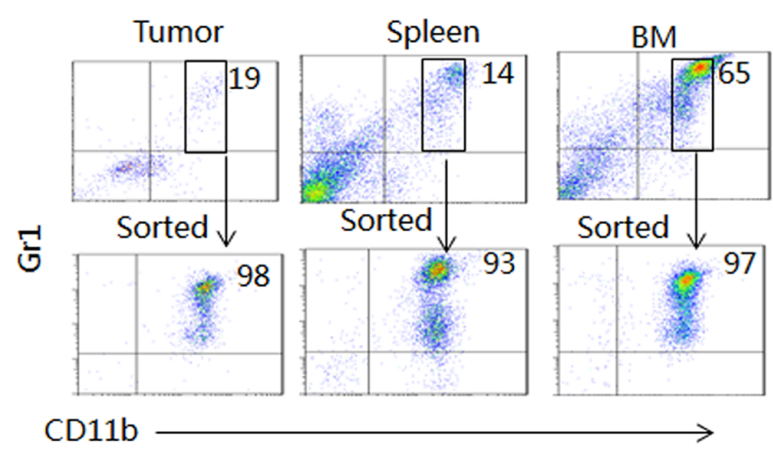

C

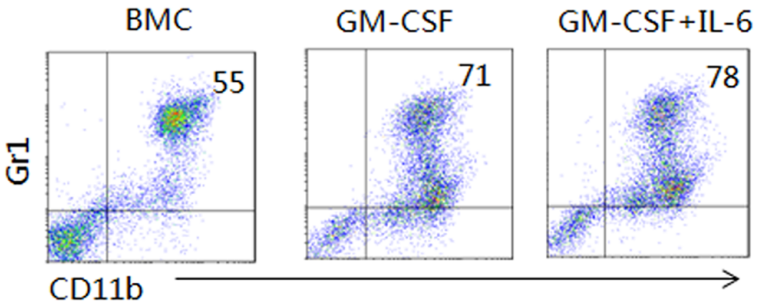

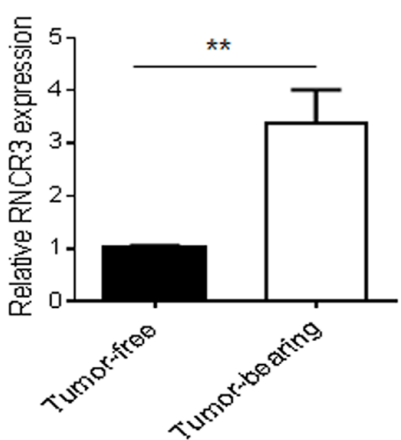
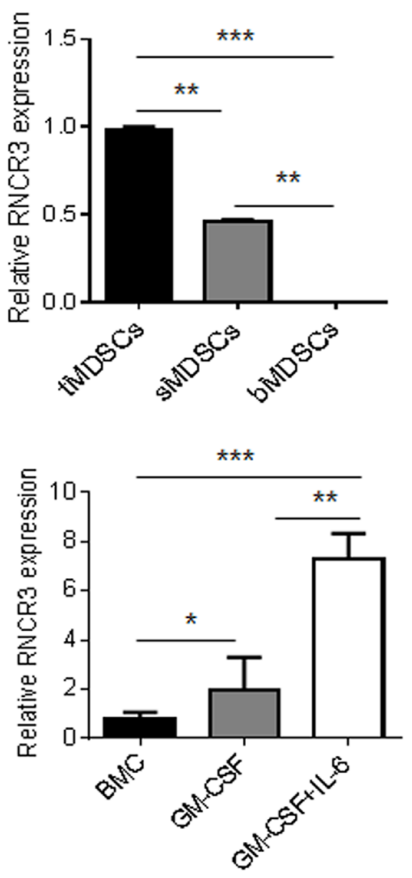

D
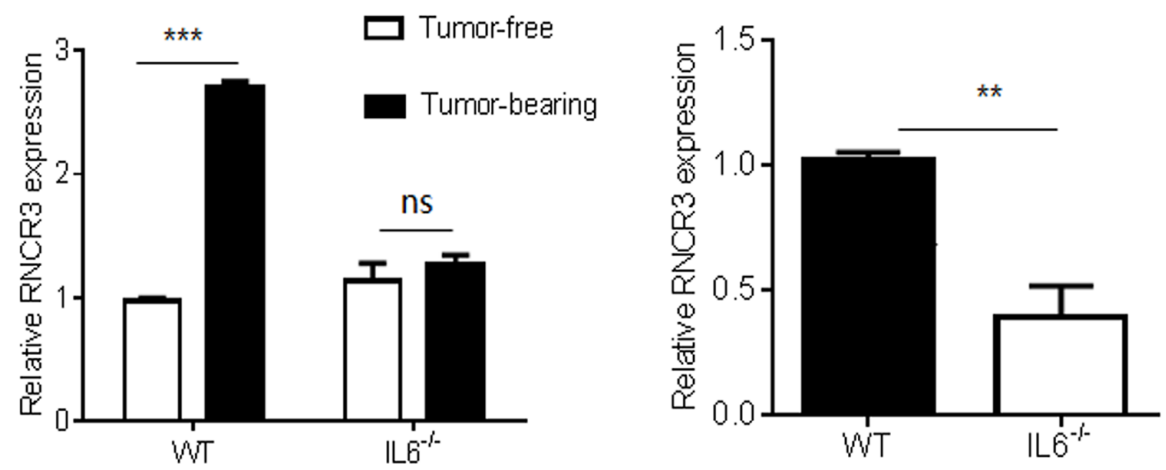

Figure 1: RNCR3 is upregulated in tumor microenvironment. (A) Flow cytometric analyses (left) and RNCR3 qRT-PCR (right)

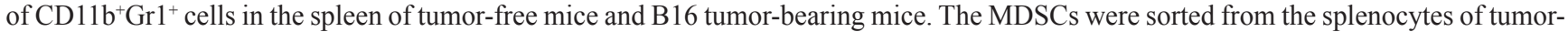
bearing mice and tumor-free mice. Iso.ctrl., antibody isotypic control. (B) Flow cytometric analyses (left) and RNCR3 qRT-PCR (right)

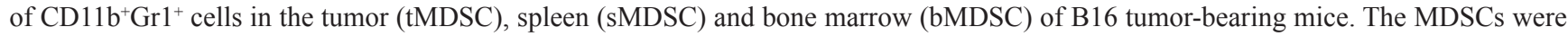
sorted from tumor tissues, spleen and bone marrow (BM) of B16 tumor-bearing mice. (C) Flow cytometric analyses (left) and RNCR3 qRTPCR (right) of CD11 b $\mathrm{Gr}^{+}{ }^{+}$MDSCs induced by GM-CSF or GM-CSF plus IL-6. Fresh CD11 $\mathrm{b}^{+} \mathrm{Gr}^{+}{ }^{+} \mathrm{BMCs}(\mathrm{BMC})$ were used as control. (D) QRT-PCR of RNCR3 in CD11 b $\mathrm{Gr}^{+}$MDSCs isolated from the BM of WT or IL-6-- tumor free and tumor-bearing mice (Left), and

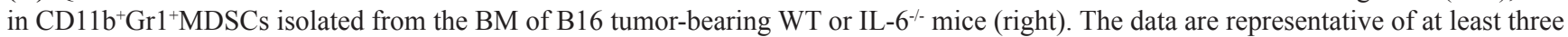
separate experiments. ${ }^{*} \mathrm{P}<0.05,{ }^{* *} \mathrm{P}<0.01,{ }^{* * *} \mathrm{p}<0.005$; Ns, no significance. 
A

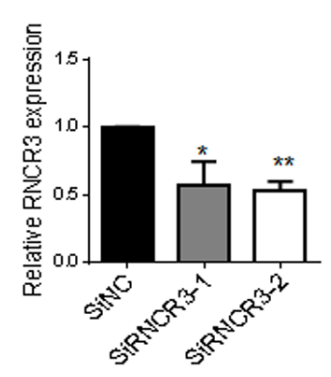

D

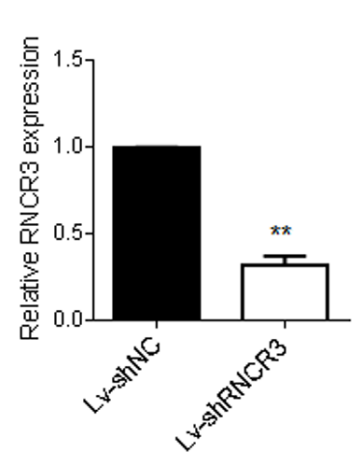

B

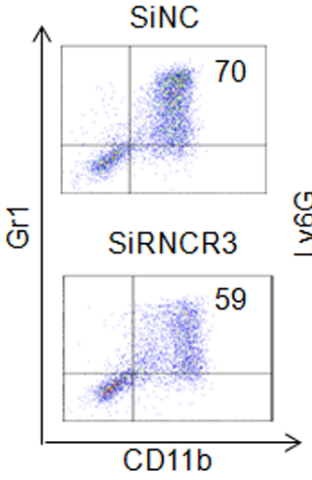

SiNC

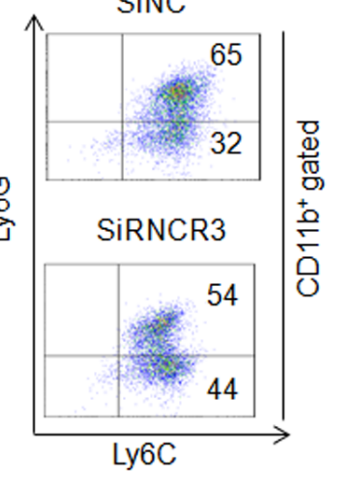

C

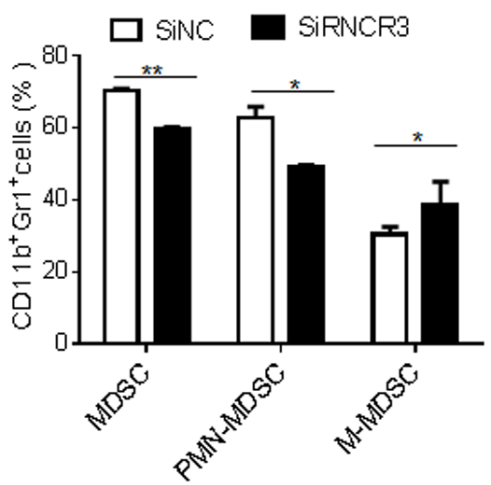

DAPI
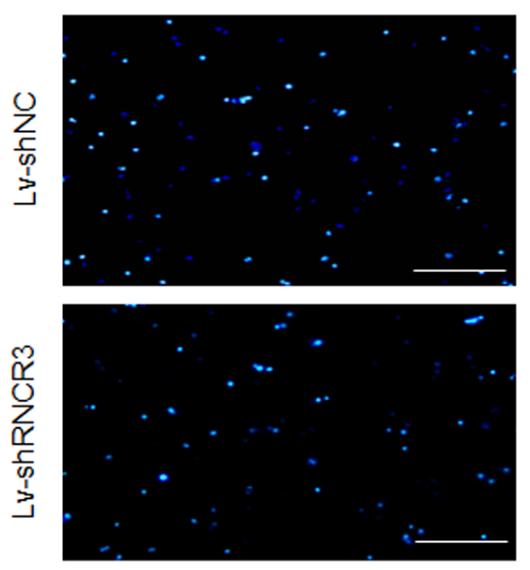

GFP
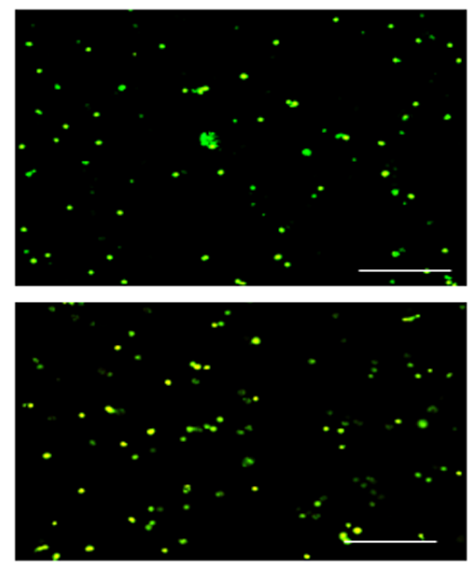

E

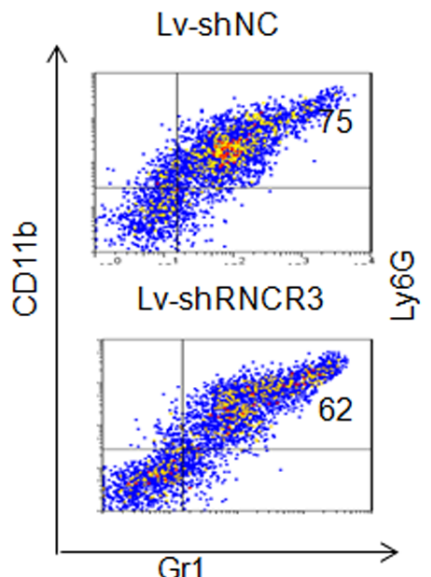

Lv-shNC

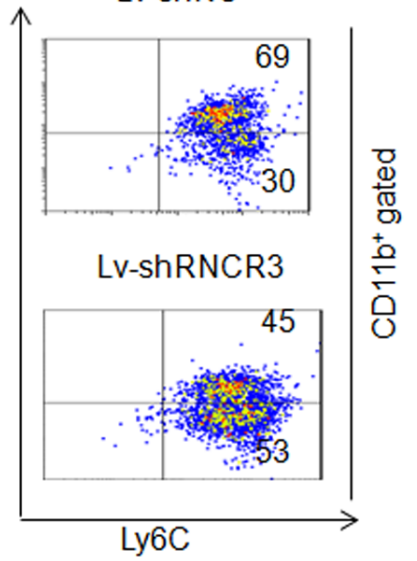

F

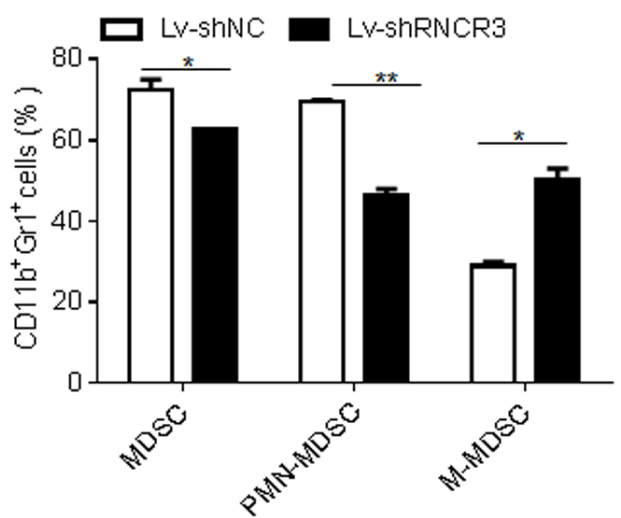

Figure 2: RNCR3 knockdown interrupts MDSC differentiation in vitro. (A) QRT-PCR of lncRNA RNCR3 in MDSCs transfected with SiNC (siRNA control), SiRNCR3-1(RNCR3 siRNA 1) or SiRNCR3-2 (RNCR3 siRNA 2). (B) Flow cytometric analyses of MDSCs transfected with SiNC or SiRNCR3 (RNCR3 siRNA 2). (C) Statistical analyses of MDSCs transfected with SiNC or SiRNCR3 (RNCR3 siRNA 2). (D) QRT-PCR of RNCR3 in MDSCs transduced with control shRNA/lentiviruses (Lv-shNC) or RNCR3 shRNA/ Lentiviruses (Lv-shRNCR3) (left), and fluorescence microscopy of lncRNA shRNA/lentiviruses transduced mouse MDSC (right). Blue (DAPI), nuclei; Green(GFP), lentivirus shRNA lentivirus. Rule bar $=100 \mu \mathrm{M}$. (E) Flow cytometric analyses of MDSCs transduced with control shRNA/lentiviruses (Lv-shNC) or RNCR3 shRNA/Lentiviruses (Lv-shRNCR3). (F) Statistical analyses of MDSCs transduced with control shRNA/lentiviruses (Lv-shNC) or RNCR3 shRNA/Lentiviruses (Lv-shRNCR3). The data are representative of at least three separate experiments. ${ }^{*} \mathrm{P}<0.05,{ }^{* *} \mathrm{P}<0.01,{ }^{* * *} \mathrm{p}<0.005$; Ns, no significance. 
2D). Similar results could be also detected in the RNCR3 shRNA/lentivirus knockdown MDSCs (Figure 2E and $2 \mathrm{~F}$ ). These findings indicate that RNCR3 may regulate the differentiation of PMN-MDSCs and M-MDSCs subsets.

\section{RNCR3 knockdown weakens immunosuppressive function of MDSCs in vitro}

We next determined the effect of RNCR3 on the immunosuppressive function of MDSCs. We cocultured OVA-specific OT-I spleen cells with RNCR3 siRNA or RNCR3 shRNA knockdown MDSCs. RNCR3 knockdown MDSCs attenuated the suppression of MDSCs on IFN- $\gamma$ secretion of OT-I T cells (Figure 3A and 3B). The suppression of MDSCs on T cells mainly depends on Arg-1, iNOS, NOX2 and their products [6, 7]. QRT-PCR and Western blotting showed that RNCR3 knockdown significantly decreased the expression of Arg-1 and iNOS in MDSCs (Figure 3C and 3D). RNCR3 silencing MDSCs had also lower levels of NO (Figure 3E and 3G) and ROS (Figure $3 \mathrm{~F}$ and $3 \mathrm{H}$ ) than control MDSCs. These results suggest that RNCR3 may regulate the immunosuppressive function of MDSCs.

\section{RNCR3 knockdown suppresses differentiation and function of MDSCs in vivo}

To further determine the effect of RNCR3 on the differentiation and function of MDSCs, we employed a murine B16 melanoma model. RNCR3 knockdown MDSCs were injected into mice after inoculating B16 tumor cells. The tumor growth was monitored at the indicated time. Mice injected with MDSCs transfected with RNCR3 siRNAs or transduced with RNCR3 shRNA/lentivirus showed decreased tumor growth, tumor size and tumor weight (Figure 4A-4F) as compared to control. In addition, we also found mice injected with MDSCs transfected with RNCR3 siRNA had a decreased
A

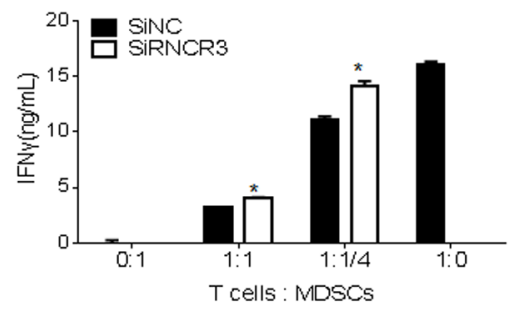

C

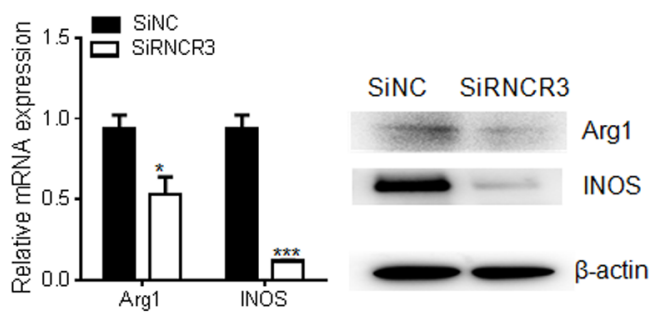

E

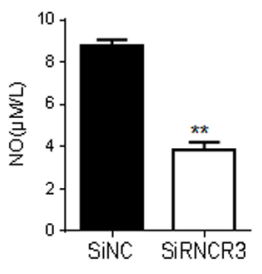

$\mathbf{F}$

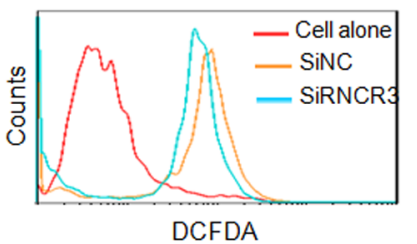

B

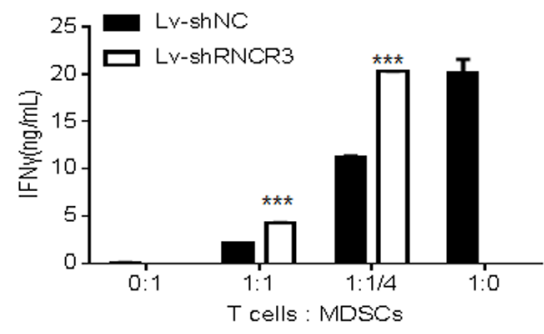

D

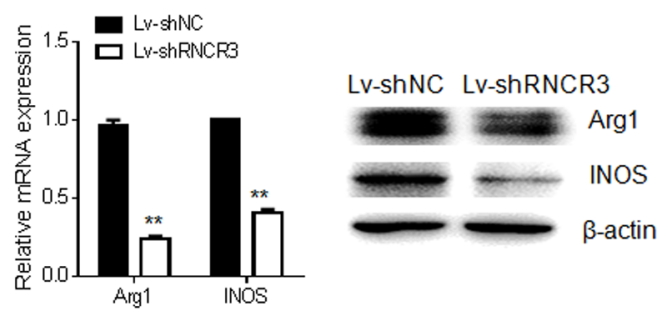

G

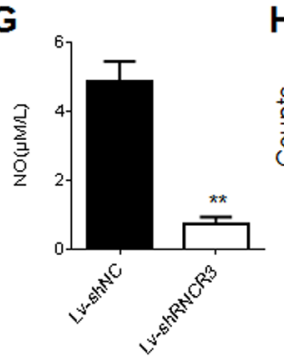

H

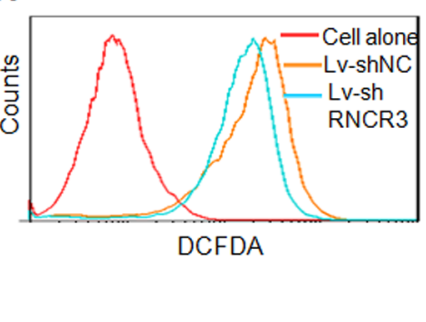

Figure 3: RNCR3 knockdown impairs immunosuppressive function of MDSCs in vitro. (A and B) Suppressive capacity of RNCR3 siRNA (SiRNCR3) transfected (A) or RNCR3 shRNA/Lentivirus (Lv-shRNCR3) transduced (B) MDSCs. Activity of T cells was measured by their capacity to produce IFN- $\gamma$ upon OVA-MHCI specific peptide stimulation. (C and D) QRT-PCR and immunoblotting of Arg-1 and iNOS in RNCR3 siRNA (SiRNCR3) transfected (C) or RNCR3 shRNA/Lentivirus (Lv-shRNCR3) transduced (D) MDSCs. (E and G) NO production in RNCR3 siRNA (SiRNCR3) transfected (E) or RNCR3 shRNA/Lentivirus (Lv-shRNCR3) transduced (G) MDSCs. (F and H) Flow cytometric analyses of ROS in RNCR3 siRNA (SiRNCR3) transfected (F) or RNCR3 shRNA/Lentivirus (LvshRNCR3) transduced (H) MDSCs. SiNC, siRNA control; Lv-shNC, control lentiviruses. The data are representative of at least three separate experiments. ${ }^{*} \mathrm{P}<0.05,{ }^{* *} \mathrm{P}<0.01,{ }^{* * *} \mathrm{p}<0.005$; Ns, no significance. 


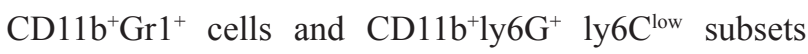
in the spleen and tumor (Figure 4G-4I), supporting the involvement of RNCR3 in the differentiation of MDSCs. The percentages of $\mathrm{CD}^{+}$and $\mathrm{CD}^{+}$cells were increased in the tumor of the mice injected with MDSCs transfected with RNCR3 siRNA as compared to control (Figure 4J), indicating the reduced suppressive ability in RNCR3 knockdown MDSCs. These changes of immune cell populations in the spleen and tumor were also found in mice injected with MDSCs transduced with RNCR3shRNA/lentiviruses (Supplementary Figure 2 ). Although MDSCs have direct cytotoxicity on the tumor cells [30], but this effect is very weak and no difference between RNCR3 knockdown and control treated MDSCs (not shown), eliminating the direct antitumor effect of RNCR3-treated MDSCs. Taken together, these results indicate that RNCR3 knockdown interrupts the differentiation of MDSCs and weakens the immunosuppressive activity of MDSCs in vivo.

\section{Regulation of RNCR3 is through a reciprocal interaction with miR-185-5p}

LncRNAs could act as ceRNAs by interacting with miRNAs to regulate cell function and differentiation. A previous study has shown that RNCR3 can function as a ceRNA by binding to miR-185-5p, and miR-185-5p mimics significantly decrease the level of RNCR3 [28]. Similar phenomenon was also observed in MDSCs. We first found that miR-185-5p could be downregulated by GM-CSF plus IL-6 (Supplementary Figure 3A), which was inverse to RNCR3 (Figure 1C), suggesting a potential correlation between RNCR3 and miR-185-5p. While miR185-5p mimics was transfected into BMCs, the level of RNCR3 was significantly downregulated (Supplementary Figure 3B and 3C); Conversely, knockdown RNCR3 increased miR-185-5p level (Supplementary Figure 3D). Since RNCR3 may interact with miR-185-5p, we next determined whether miR-185-5p was involved in the MDSC differentiation. BMCs were transfected with miR-185-5p mimics or miR-185-5p inhibitor in the presence of GM-CSF and IL-6. The level of miR185-5p was significantly higher in MDSCs transfected with miR-185-5p mimics (Figure 5A). After induction for 4 days, miR-185-5p mimics reduced the percentage

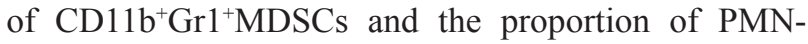
MDSCs, but the proportion of M-MDSCs in CD11b cells increased as compared to those transfected with mimics control (Figure 5B and 5C). Furthermore, mRNA and protein levels of Arg-1 and iNOS also decreased in those MDSCs transfected with miR-185-5p mimics (Figure 5D and $5 \mathrm{E}$ ). In contrast, miR-185-5p inhibitor significantly downregulated the level of miR-185-5p (Figure 5F), increased the percentage of $\mathrm{CD} 11 \mathrm{~b}^{+} \mathrm{Gr} 1^{+} \mathrm{MDSCs}$ and PMN-MDSCs, and decreased M-MDSCs as compared to those transfected with inhibitor control (Figure 5G and $5 \mathrm{H})$. MiR-185-5p inhibitor transfected MDSCs also increased Arg-1 and iNOS (Figure 5I and 5J). Thus, our data demonstrate that miR-185-5p may regulate the differentiation and function of MDSCs. We next further determined whether RNCR3 regulated the differentiation of MDSCs by binding to miR-185-5p. We found that miR-185-5p inhibitor could partially recover reduced MDSCs and PMN-MDSCs, which was caused by RNCR3 knockdown (Figure $5 \mathrm{~K}$ and $5 \mathrm{~L}$ ), further supporting that miR-185-5p may interact with RNCR3 to affect the differentiation and function of MDSCs. Taken together, these results demonstrate that RNCR3-mediated differentiation and function of MDSCs is through miR185-5p.

\section{miR-185-5p directly targets Chop}

We next investigated how miR-185-5p to regulate the differentiation and function of MDSCs. Generally, miRNAs regulate cell processes by controlling the expression of their target genes. We first predicted the target gene(s) of miR-185-5p by Targetscan and miRanda, and found that Chop was a potential target gene (Figure 6A). To demonstrate that Chop is a target of miR-1855p, we constructed luc-Chop 3'UTR and luc-Chop 3'UTR mutant (mutated on the putative miR-185-5p sites) vectors (Figure 6A). Luciferase assays showed that miR-185-5p mimics inhibited luciferase activity of luc-Chop 3'UTR but not luc-Chop 3'UTR mutants (Figure 6B), suggesting that miR-185-5p may bind with Chop 3'UTR. Further studies showed that miR-185-5p mimics also inhibited the expression of Chop (Figure 6C); whereas miR-185-5p inhibitor promoted the expression of Chop (Figure 6D). Thus, we demonstrate that Chop is a target of miR-185-5p.

Chop is the target of miR-185-5p, and miR-185$5 \mathrm{p}$ regulates MDSC differentiation, so we proposed that Chop knockdown should exert the same effect on MDSC differentiation with miR-185-5p mimics. Previous study show that Chop may promote differentiation and function of MDSCs in vivo [30]. We also found that Chop knockdown resulted in a decrease in the percentage of $\mathrm{CD} 11 \mathrm{~b}^{+} \mathrm{Gr} 1^{+} \mathrm{MDSC}$ as compared to control (Figure 7A and 7B). Furthermore, Chop knockdown also caused a significant decrease in the proportion of PMN-MDSCs and increase in M-MDSCs (Figure 7C). Meanwhile, qPCR revealed that Chop knockdown decreased the expression of Arg-1 and iNOS (Figure 7D). These results show that the effects of miR-185-5p on MDSCs are through targeting Chop.

\section{Regulation of RNCR3 in MDSCs is through upregulating Chop by sponging miR-185-5p}

We finally investigated the relationship among lncRNA RNCR3, miR-185-5p and Chop in MDSCs. Since RNCR3 could act as ceRNAs interacting with miR-185-5p 
A

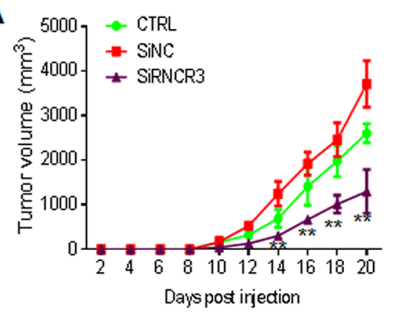

D

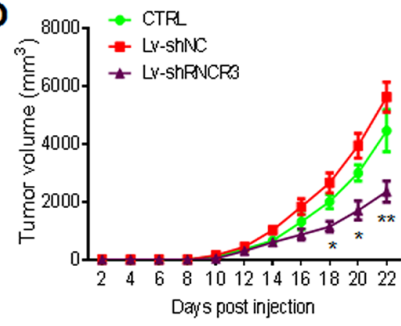

B

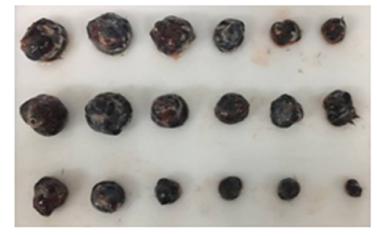

E

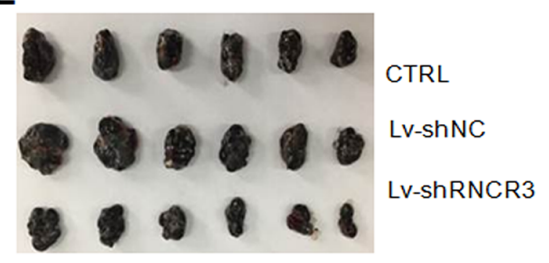

G

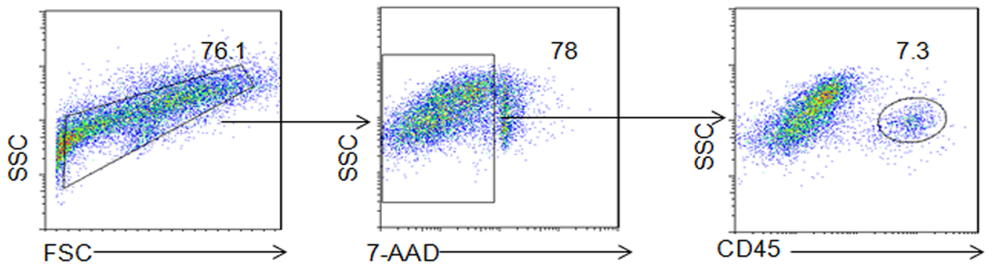

C

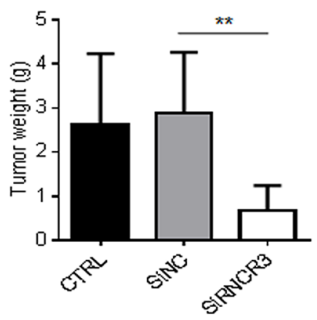

$\mathbf{F}$

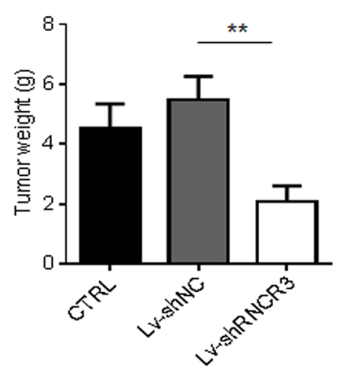

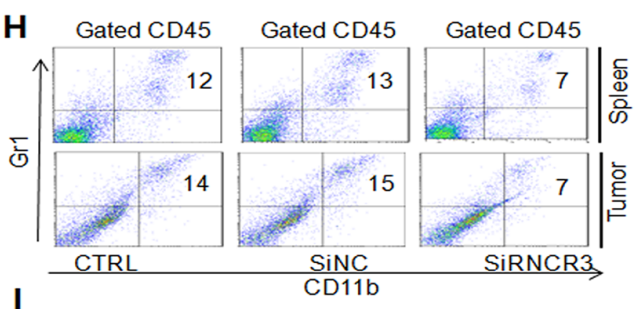

I
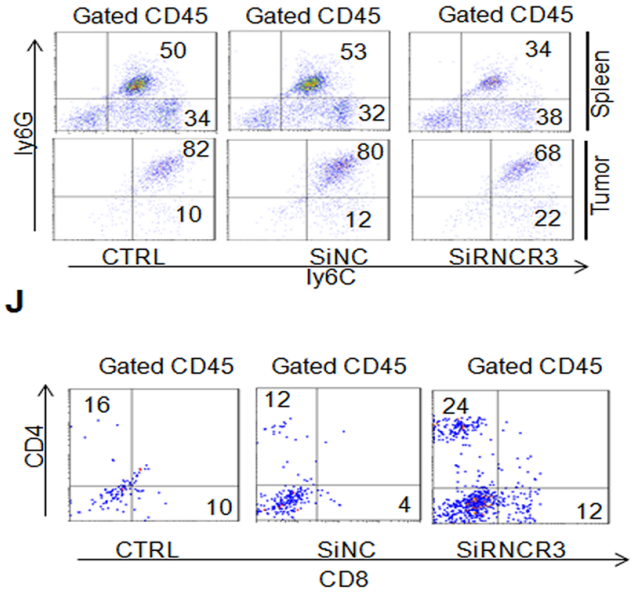
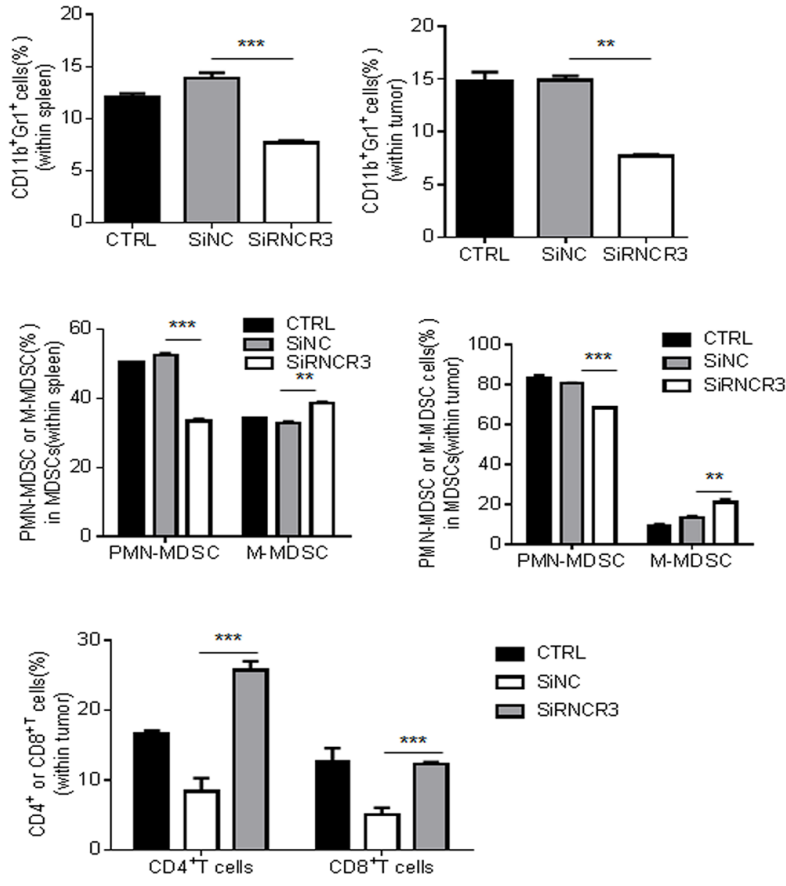

Figure 4: RNCR3 knockdown affects differentiation and immunosuppressive function of MDSCs in vivo. (A-F) Tumor growth (A, D), tumor size (B, E) and tumor weight $(C, F)$ in C57/BL6 mice bearing B16 tumors $(N=6)$, which were injected with CD45.1 $1^{+}$MDSCs transfected with SiRNCR3 (RNCR3 siRNA) (A, B, C) or transduced by shRNA (Lv-shRNCR3) (D, E, F). SiNC, siRNA control; Lv-shNC, control lentivirus; CTRL, without MDSC injection. (G) Gated strategies for tumor immune cells. (H and I) Flow cytometric and statistical analyses of MDSCs (H) and their subpopulations (I) in the spleen and tumor from mice injected with CD45.1 $1^{+}$ MDSCs transfected with SiNC or SiRNCR3. (J) Flow cytometric and statistical analyses of $\mathrm{CD}^{+}$or $\mathrm{CD}^{+}$cells in tumor from mice injected with or without CD45.1 $1^{+} \mathrm{MDSCs}$ transfected with SiNC or SiRNCR3. The data are from three separate experiments. ${ }^{*} \mathrm{P}<0.05,{ }^{* * *} \mathrm{P}<0.01$, *** $<0.005$; Ns, no significance. 
A
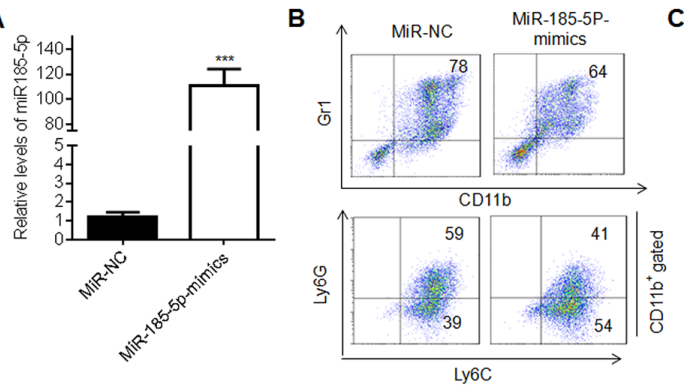

MiR-NC
口iR-185-5P-mimics

D

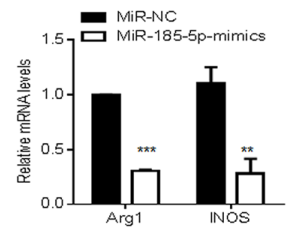

E
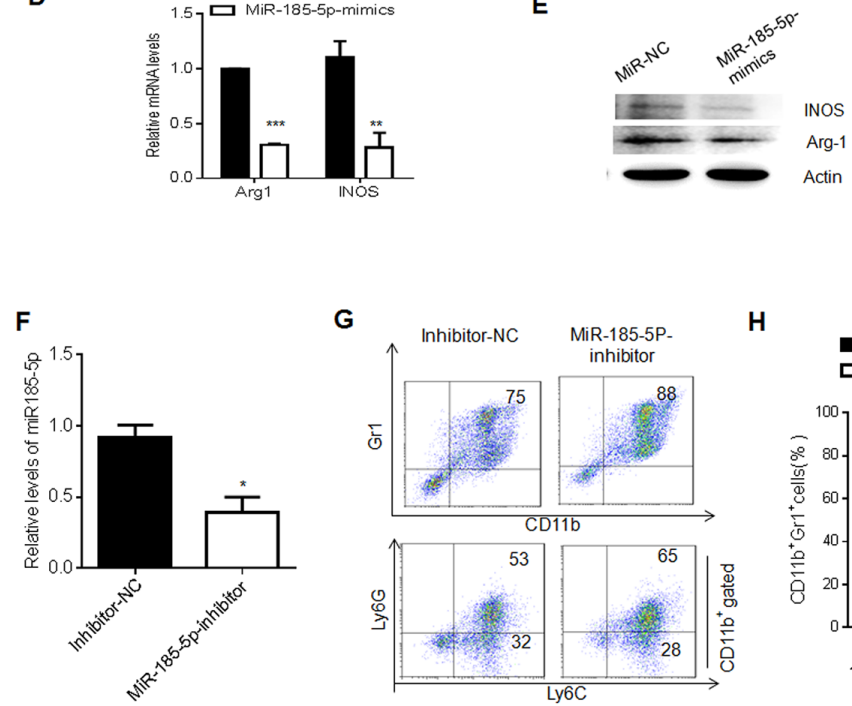

G

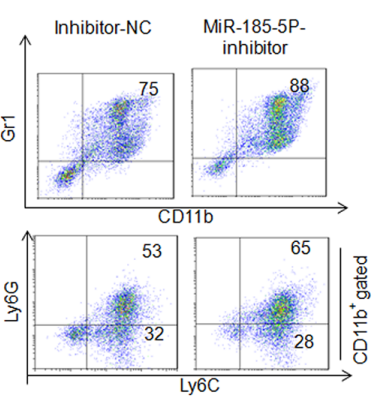

H

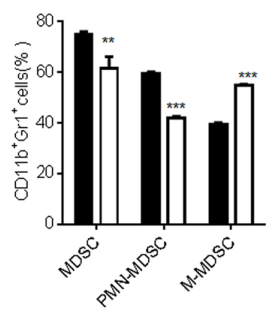

INOS

Actin

I

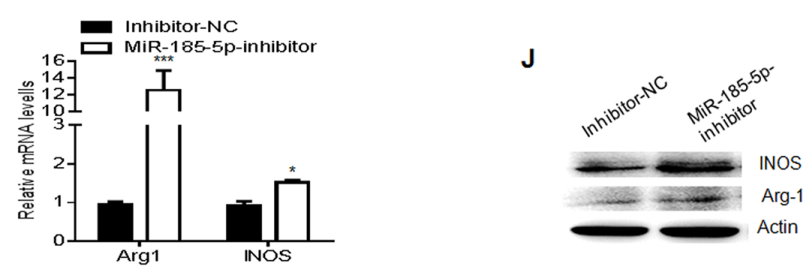

K

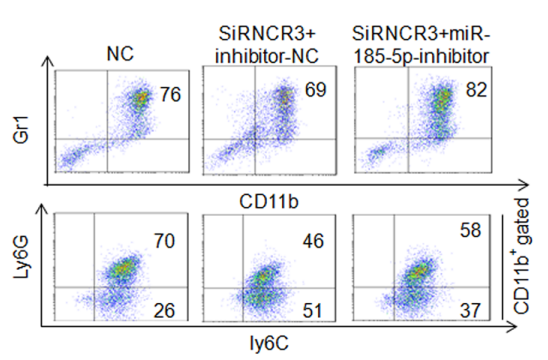

L
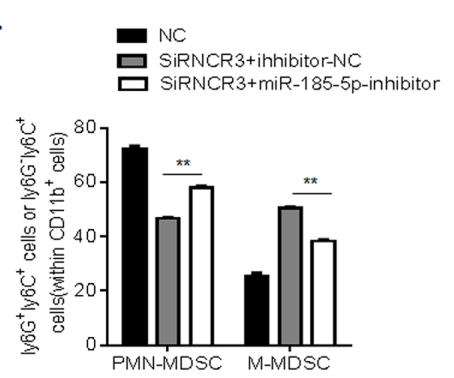

Figure 5: MiR-185-5p regulates differentiation and function of MDSCs. (A) QRT-PCR of miR-185-5p in MDSCs transfected with miR-185-5p mimics or MiR-NC (mimics control) in the presence of GM-CSF and IL-6. (B and C) Flow cytometric (B) and statistical analyses (C) of the MDSCs transfected with miR-185-5p mimics or NC. (D and E) QRT-PCR (D) and Western blot (E) of iNOS and Arg-1 in MDSCs transfected with miR-185-5p mimics control (NC) or miR-185-5p mimics. (F) QRT-PCR of miR-185-5p in the MDSCs transfected with miR-185-5p inhibitor or inhibitor control (NC). (G and $\mathbf{H})$ Flow cytometric (G) and statistical analyses (H) of MDSCs transfected with miR-185-5p inhibitor or inhibitor control (NC). (I and J) QRT-PCR (I) and Western blot (J) of iNOS and Arg-1 in MDSCs transfected with miR-185-5p inhibitor control (NC) or miR-185-5p inhibitor. (K and $\mathbf{L})$ Flow cytometric (K) and statistical analyses (L) of MDSCs transfected with RNCR3 siRNA and miR-185-5p inhibitor (siRNCR3+miR-185-5p inhibitor) or inhibitor control (siRNCR3+inhibitor-NC). NC, siRNA and inhibitor control. The data are representative of at least three separate experiments. ${ }^{*} \mathrm{P}<0.05$, ${ }^{* *} \mathrm{P}<0.01,{ }^{* * *} \mathrm{p}<0.005 ; \mathrm{Ns}$, no significance. 
during MDSC differentiation, and Chop is a target of miR$185-5 \mathrm{p}$, it is possible for RNCR3 to affect the expression of Chop. Indeed Chop was significantly downregulated in MDSCs transfected with RNCR3 siRNA as compared to control (Figure 8A). Importantly, Chop downregulated by silencing RNCR3 in MDSCs was rescued by miR-185-5p inhibitor (Figure $8 \mathrm{~B}$ ), indicating that RNCR3 positively regulates Chop expression by inhibiting miR-185-5p. To further illustrate the relationship among RNCR3, miR-185-5p and Chop, we examined the expression of RNCR3, miR-185-5p and Chop in differently isolated MDSCs (Figure 8C-8E), and different factors mediated MDSCs (Figure 8F-8H). While the level of RNCR3 expression was high, miR-185-5p was low and Chop was high; Conversely, while RNCR3 was low, the expression of miR-185-5p was high and Chop's level was low (Figure $8 \mathrm{C}-8 \mathrm{H})$. Since both RNCR3 and Chop RNAs are targets of miR-185-5p, it also is necessary to determine whether miR-185-5p has higher affinity to RNCR3 than to Chop mRNA. To test this, we first detected the Chop mRNA and RNCR3 expression dynamics in response to miR-185$5 \mathrm{p}$ transfection. While MDSCs from bone marrow were transfected miR-185-5p mimics, the levels of Chop and RNCR3 were both downregulated in a time-dependent and dose-dependent manner; but the alterations of RNCR3 was much remarkable than Chop, implying that miR-185-5p may have higher affinity to RNCR3 than to Chop mRNA
(Supplementary Figure 4A and 4B). To further prove this, we employed dual luciferase report assays. RNCR3 WT (wild-type) and MUT (mutated) dual-luciferase reporter were constructed. While $293 \mathrm{~T}$ cells were transfected with miR-185-5p mimics, luc-RNCR3 had lower luciferase activity than luc-Chop, indicating that miR-185-5p has higher affinity to RNCR3 (Supplementary Figure 4C4E). Finally, RNA fluorescence in situ hybridization also showed that RNCR3 located not only in the nucleus but also in the cytoplasm of MDSCs (Figure 8I), further indicating the possibility for RNCR3 to bind with miR$185-5 \mathrm{p}$ in the cytoplasm. Taken together, our data suggest a RNCR3/miR-185-5p/Chop autologously strengthening pathway for the differentiation and immunosuppressive function of MDSCs.

\section{DISCUSSION}

In this study we first found that MDSCs may express lncRNA RNCR3, and the expression is upregulated by tumor associated factors. We demonstrate that RNCR3 may promote MDSC differentiation and immunosuppressive function of MDSCs by binding to miR-185-5p and thereby releasing Chop. Since the levels of RNCR3 expression in MDSCs may be regulated by tumor-associated factors, this may disclose how MDSCs autologously strengthen differentiation and function
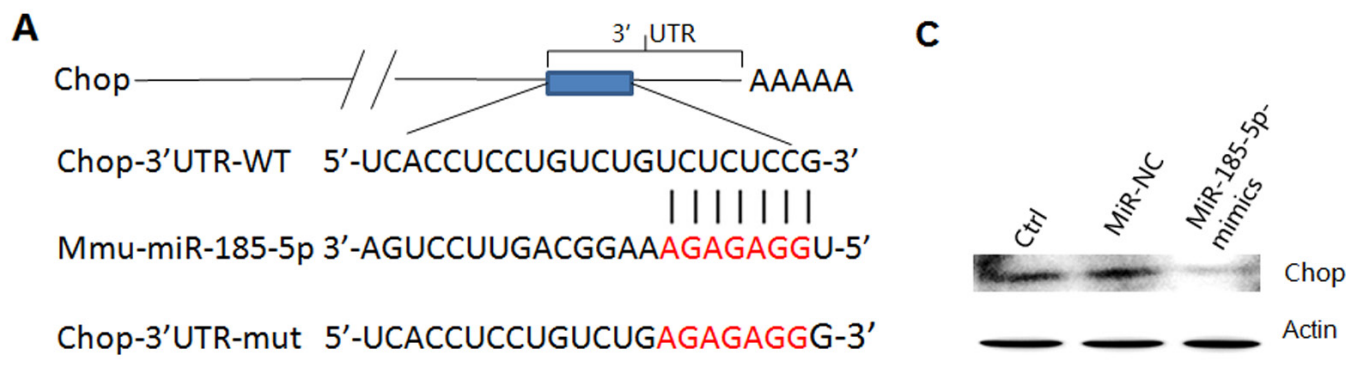

B
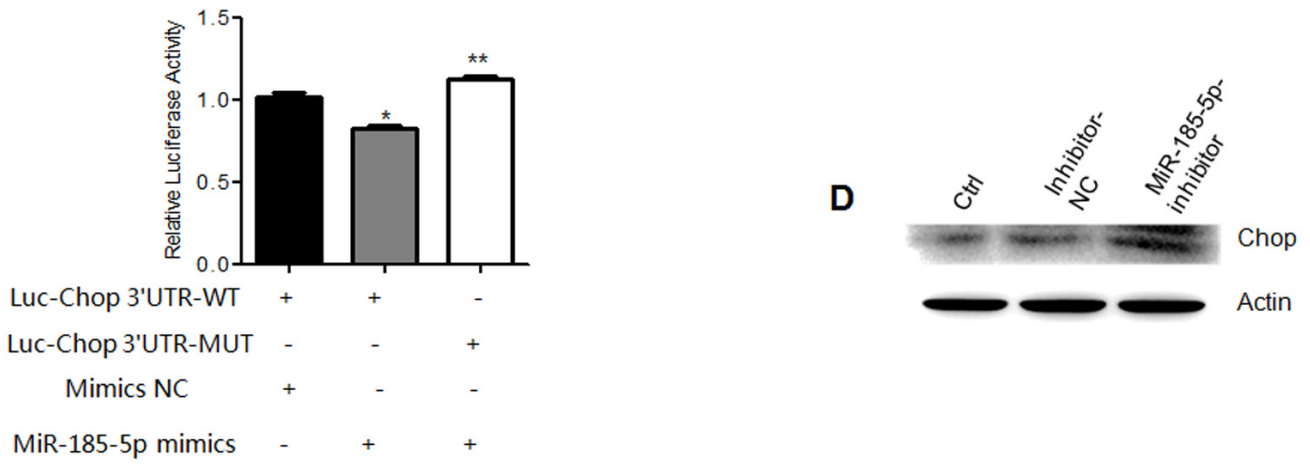

Figure 6: MiR-185-5p directly targets Chop. (A) Predicted paring of miR-185-5p to the 3'-UTR of Chop and the mutant site (Red) of Chop 3'-UTR in pSiCHECK ${ }^{\mathrm{TM}}$-2 luciferase reporter vector. (B) Dual-luciferase reporter assay of 293T cells co-transfected with Chop 3'-UTR-WT or Chop 3'-UTR-Mut and miR-185-5p mimics or mimics control (NC). (C) Western blot of Chop in MDSCs transfected with mimics control (miR-NC) or miR-185-5p mimics. Untransfected MDSCs as control. (D) Western blot of Chop in MDSCs transfected with inhibitor control (inhibitor-NC) or miR-185-5P inhibitor. Untransfected MDSCs as control. ${ }^{*} \mathrm{P}<0.05,{ }^{* *} \mathrm{P}<0.01,{ }^{* * *} \mathrm{p}<0.005$; Ns, no significance. 
of MDSCs in the tumor environment. Thus, while tumor-associated factors promote differentiation and immunosuppressive function of MDSCs, the upregulated RNCR3 may further promote the development of these processes.

More and more evidences have shown that lncRNAs play important roles in directing the development and differentiation of diverse immune cells. Multiple lincRNAs have been identified in myeloid immune cells such as that lncRNA HOTAIRM1 is reported to regulate the differentiation of myeloid cells [21]; Morrbid regulates the lifespan of short-lived myeloid cells [22]; Lnc-DC regulates differentiation of human dendritic cells [23] and lincRNA-Cox 2 can turn on and off distinct classes of immune genes by different protein partners [31]. However, the effect of lncRNA on MDSCs is still absence of reports. We here demonstrate that lncRNA RNCR3 not only promotes the differentiation of MDSCs but also strengthens the immunosuppressive function of MDSCs.

LncRNAs regulate cell differentiation by multitudinous mechanisms such as lncRNAs can function as a ceRNA by sponging microRNAs or function as a guide, scaffold or decoy molecule by interacting with its protein partner [32]. LncRNAs bind to and sequester specific miRNA(s) to protect the target mRNAs from repression, which are revealed in many processes, including cell differentiation, tumorigenesis and pluripotency [33]. MicroRNA, a post-transcriptional regulator can repress target gene expression by binding to its complementary sequences in 3' untranslated regions (3'UTRs) [34]. CeRNAs usually own miRNA-response elements and prevent the corresponding mRNAs from being degraded by competing for miRNAs. LncND, which contains 16 microRNA response elements for miR-143$3 p$, regulates the expression of Notch-1 and Notch- 2 by sequestering miR-143-3p [35]. HOTAIR is reported to act as a ceRNA by sponging miR-613 and then suppresses its expression, leading to affect the expression of notch 3 in pancreatic cancer [36]. RNCR3 functions as a ceRNA by sponging miR-185-5p and regulates the levels of KLF2 in endothelial cells [28]. We here also found that RNCR3 may functions as a ceRNA to promote Chop expression by sponging miR-185-5p during MDSC differentiation.

Chop is a cellular stress sensor C/EBP-homologous protein, which is induced by tumor-linked reactive oxygen and nitrogen species. Chop plays an important role in
A

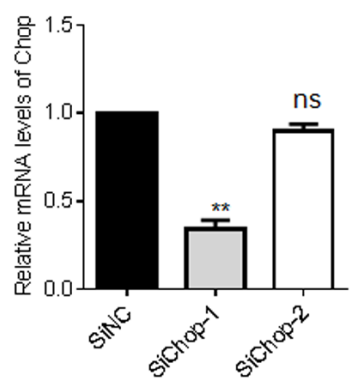

B

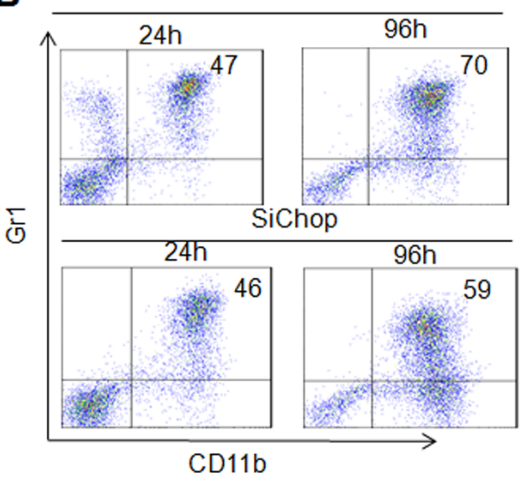

C

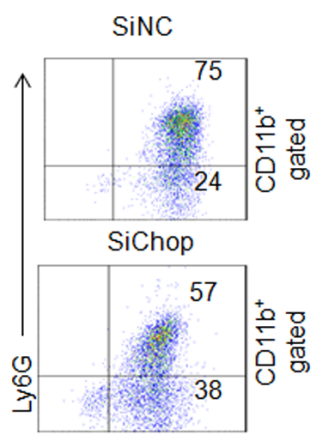

Ly6C

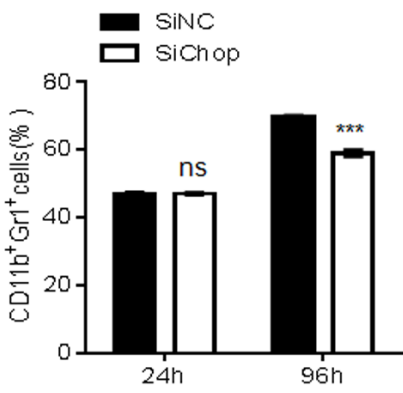

D
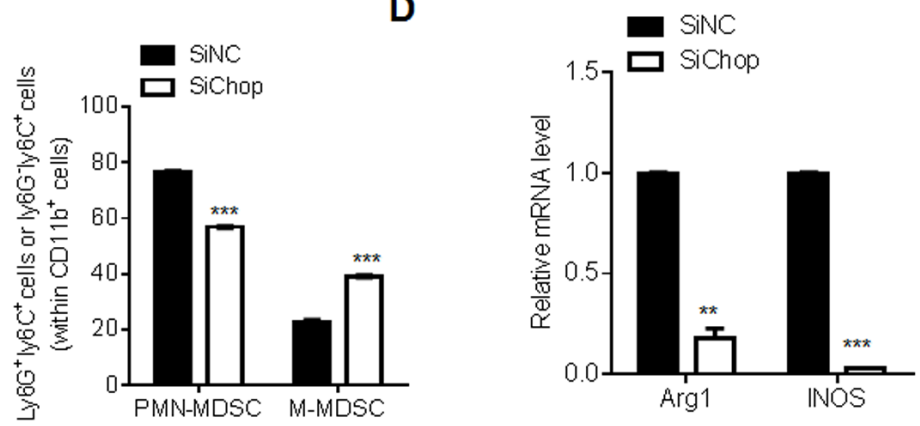

Figure 7: Chop knockdown exerts the same effect to miR-185-5p mimics during MDSC differentiation. (A) QRT-PCR of Chop in MDSCs transfected with siNC (control siRNA), siChop-1(Chop siRNA 1) or siChop-2 (Chop siRNA 2). (B) Flow cytometric and statistical analyses in $\mathrm{CD} 11 \mathrm{~b}^{+} \mathrm{Gr} 1^{+}$MDSCs transfected with siNC or siChop. (C) Flow cytometric and statistical analyses of MDSC subpopulations in MDSCs transfected with siNC and siChop. (D) QRT-PCR of Arg1 and iNOS in MDSCs transfected with siNC and siChop. The data are representative of at least three separate experiments. ${ }^{*} \mathrm{P}<0.05,{ }^{* *} \mathrm{P}<0.01,{ }^{* * *} \mathrm{p}<0.005$; Ns, no significance. 
regulating the accumulation and immunosuppressive function of MDSCs. Chop-deficient MDSCs may display reduced signaling through CCAAT/enhancerbinding protein- $\beta$ (cEBP $\beta$ ) and reduced phospho-STAT3 [30]. Previous studies have shown that $\mathrm{EBBP} \beta$ and STAT3 are critical transcription factors in regulating the differentiation and function of MDSCs [37-39]. Ablation of STAT3 expression through the use of conditional knockout mice or selective STAT3 inhibitor markedly reduces the expansion of MDSC and increased $\mathrm{T}$ cell responses in tumor-bearing mice $[40,41]$. Recent studies also found that the generation of $\mathrm{Ly}^{-} \mathrm{C}^{-}$monocytes involves induction of the transcription factor $\mathrm{C} / \mathrm{EBP} \beta$ and $\mathrm{C} / \mathrm{EBP} \beta$-deficient mice lack Ly6C- monocytes [38]. MDSCs lacking Chop have also decreased immuneregulatory functions and show the ability to prime $\mathrm{T}$ cell function and induce antitumor responses [30]. Our results also exhibit that Chop knockdown may affect the differentiation of MDSCs and decrease the levels of Arg-1 and iNOS, which plays a critical role in inhibiting $\mathrm{T}$ cell immunity. Meanwhile, we also found that Chop is significantly regulated by miR-185-5p, which can target multiple targeting molecules such as KLF2 or ABCC1 $[28,42]$. Since miR-185-5p may be sponged by lncRNA RNCR3 during MDSC differentiation, we disclose a RNCR3/miR-185-5p/Chop regulatory network for strengthening differentiation and function of MDSCs.

MDSCs are immunosuppressive cells, which can promote tumor growth, differentiation and metastasis in a variety of ways [3]. Our studies show that adoptive transfer of MDSCs transfected with RNCR3 siRNA significantly reduces the generation of MDSCs, weakens the inhibitory function of MDSCs and suppresses tumor growth. Since RNCR3 expression may be regulated through tumor environment and inflammatory factors, our data suggest a new potential therapeutic target in cancer.

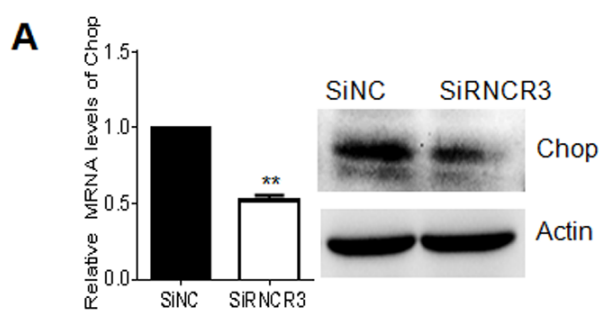

B

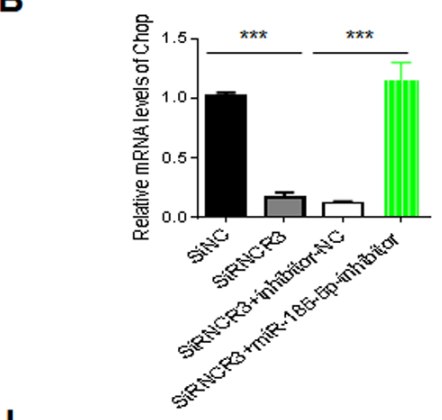

I

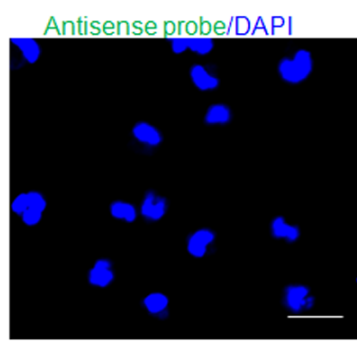

\section{C}
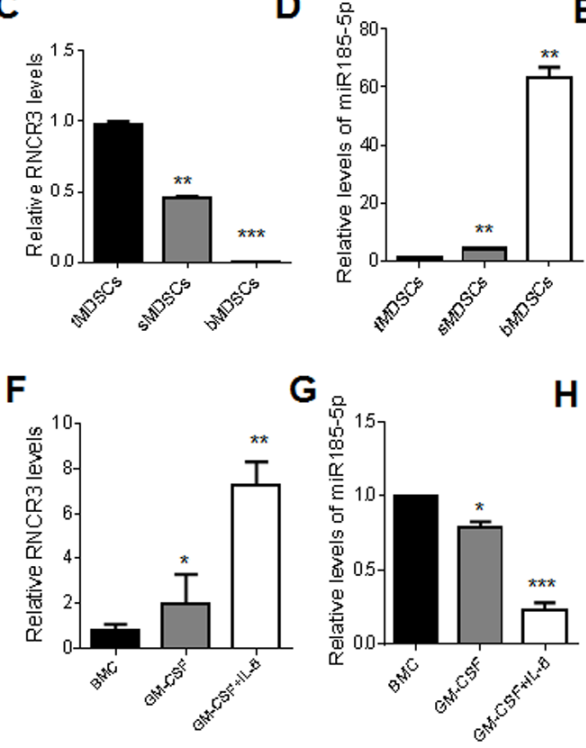

RNCR3 probe/DAPI
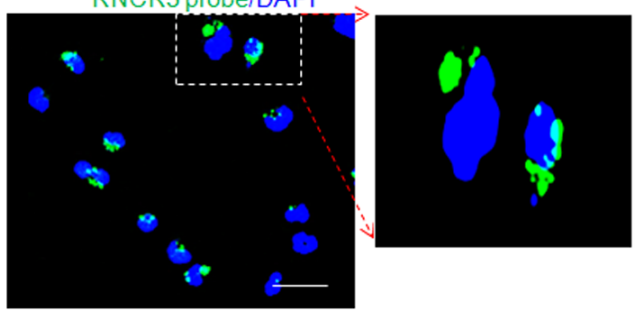

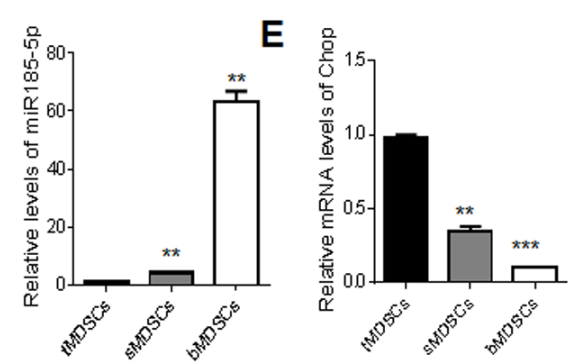

H

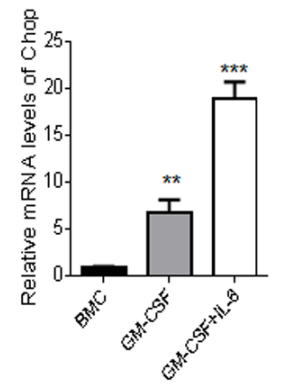

BMC GM-CSF GM-CSF + IL-6

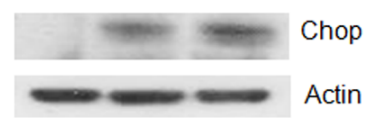

Figure 8: Regulation of RNCR3 in MDSCs is through upregulating Chop by sponging miR-185-5p. (A) QRT-PCR (left) and Western blot (right) of Chop in MDSCs transfected with siRNA control (SiNC) and RNCR3 siRNA (SiRNCR3). (B) qRT-PCR of Chop in MDSCs transfected with RNCR3 siRNA and miR-185-5p inhibitor (siRNCR3+miR-185-5p) or miR-185-5p inhibitor control (siRNRC3+NC). siNC, siRNA control. (C, D, E) QRT-PCR of RNCR3, miR-185-5p and Chop in the MDSCs isolated from the tumor tissues (tMDSCs), spleen (sMDSCs) and BM (bMDSCs) of mice bearing B16 tumor. (F, G, H) QRT-PCR of RNCR3, miR-185-5p and Chop in CD11 b $\mathrm{Gr}^{+} \mathrm{BMCs}$ (BMC), GM-CSF induced MDSCs (GM-CSF) and GM-CSF plus IL-6 mediated MDSCs (GM-CSF+IL-6). Western blot of Chop (lower in H). (I) RNA-FISH in the isolated MDSCs from the spleens of mice bearing B16 tumor. Green, RNCR3; Blue, nuclei. Rule bar $=20 \mu \mathrm{M}$. The data are representative of at least three separate experiments. ${ }^{*} \mathrm{P}<0.05,{ }^{* *} \mathrm{P}<0.01,{ }^{* * *} \mathrm{p}<0.005$; Ns, no significance. 


\section{MATERIALS AND METHODS}

\section{Mice and cells}

C57BL/6 and BALB/c mice were purchased from Beijing Animal Center (Beijing, China) and maintained in a specific pathogen-free (SPF) and controlled environment. B6.129S6-Il- $6^{\text {tmlKopf }}\left(\right.$ IL- $6^{-/}$) and CD45.1 mice were purchased from Model Animal Research Center of Nanjing University (MARC, Nanjing, Jiangsu, China). OT-I transgenic mice were provided by Dr. Linrong Lu in Zhenjiang University. Experiments were carried out using age- and gender- matched mice. All the procedures were conducted according to Institutional Animal Care and Use Committee of the Model Animal Research Center. Animal experiments were approved by the Institute's Animal Ethics Committee of Nankai University.

Murine melanoma B16 was obtained from American Type Culture Collection (ATCC, Manassas, VA, USA). Mosec ovarian cancer cells were gifted from Richard B. S. Roden (Johns Hopkins University, Maryland, USA). All of the cells were cultured in RPMI-1640 with $10 \%$ FBS (Hyclone, Logan, UT, USA) at $37^{\circ} \mathrm{C}$ in a humidified 5\% $\mathrm{CO}_{2}$ atmosphere. $\mathrm{CD} 11 \mathrm{~b}^{+} \mathrm{Gr} 1^{+}$cells were sorted by FAScan or isolated using $\mathrm{CD} 11 \mathrm{~b}$ and Gr1 MACS MicroBeads and cell isolation kit (Miltenyi Biotec, Bergisch Gladbach, Germany) according to the manufacture's instructions.

For in vitro induction of MDSCs, BMCs were obtained from the femurs of C57BL/6 mice and cultured in RPMI-1640 medium supplemented with GM-CSF (40 $\mathrm{ng} / \mathrm{ml})$ only or GM-CSF $(40 \mathrm{ng} / \mathrm{ml})$ plus IL-6 $(40 \mathrm{ng} / \mathrm{ml})$ for 4 days.

\section{Preparation of MDSCs tranfected by microRNA mimics, inhibitor and siRNA}

Murine bone marrow cells were first transfected with control siRNA, RNCR3 siRNA, Chop siRNA, miR185-5p mimics, miR-185-5p inhibitor or normal control using HiPerFect transfection reagent (Qiagen, Valencia, CA, USA) according to the manufacturer's instructions, and then cultured for 4 days in the presence of GM-CSF or GM-CSF plus IL-6. All microRNA, siRNAs and control siRNAs were purchased from Riobio (Guangzhou, China). The target sequences for RNCR3 siRNAs: siRNA1, 5'GGATGCGGGAGAACAAAGA-3' and siRNA 2, 5'TCACAGCGGACCTTGATTT-3'; The target sequences for Chop siRNAs: siRNA 1, 5'- CCTAACACGTCGATTATAT-3'; and siRNA 2, 5'- GCTCTCCAGATTCCAGTCA-3'.

\section{Construction and transduction of RNCR3 shRNA/lentivirus}

A shRNA target (5'- TCACAGCGGACCTTG ATTT-3') was chosen from the target sequences produced by BLOCK-iT ${ }^{\mathrm{TM}}$ RNAi Designer (Invitrogen) and/or by
i-Score Designer38. The shRNA constructs were made using pGreenPuro ${ }^{\mathrm{TM}}$ shRNA Cloning and Expression Lentivector kit (System Biosciences Inc.) according to the manual. The control shNC is the Luciferase Control shRNA from the kit. For packaging of lentivirus particles, the shRNA lentivector together with pMD2.G and psPAX2 packaging plasmids were cotransfected into 293T cells.

MDSCs were infected with the lentiviral supernatants in the presence of $8 \mu \mathrm{g} / \mathrm{ml}$ polybrene (Millipore) by centrifugation and then cultured with complete medium for 24 hours. The cells were then washed and cultured under GM-CSF or GM-CSF plus IL-6 for 4 days.

\section{In vitro MDSC suppression assay}

To measure immunosuppressive function of different treated MDSCs, MDSCs and splenocytes obtained from OT-I mice were co-cultured with $200 \mathrm{nM}$ OVA peptide (OVA257-264) in 96-well plates at a ratio of 1:0,1:1,1:4 and $0: 1$ for $48 \mathrm{~h}$. The production of IFN- $\gamma$ was measured by ELISA Kit (4A Biotech, Beijing, China) according to the manufacturer's instructions.

\section{Reactive oxygen species and nitric oxide detection}

Oxidation-sensitive dye DCFDA (Diacetyldichlorofluorescein, Molecular Probes/ Invitrogen) was used to measure reactive oxygen species (ROS) production by MDSCs according to the reported protocol [43]. Isolated $\mathrm{CD}_{1} 1 \mathrm{~b}^{+} \mathrm{Gr} 1^{+} \mathrm{MDSCs}$ were simultaneously cultured with $2.5 \mu \mathrm{M}$ DCFDA and $30 \mathrm{ng} /$ $\mathrm{ml}$ phorbol myristate acetate (PMA, Sigma-Aldrich) for $30 \mathrm{~min}$. Analyses were then conducted by flow cytometry as described above.

For nitric oxide production, the total nitric oxide in the cell lysate was measured using the Nitrate/Nitrite Assay Kit. Equal volumes of cell lysate $(60 \mu \mathrm{l}), 2 \mathrm{mM}$ NADPH (5 ul), FAD (10ul) and Nitrate Reductase (5ul) were incubated at $37^{\circ} \mathrm{C}$ for $30 \mathrm{~min}$, followed by addition of $10 \mathrm{ul}$ of LDH Buffer and LDH solution. After incubation for $30 \mathrm{~min}$ at $37^{\circ} \mathrm{C}$. Then, addition of $50 \mathrm{ul}$ of Griess Reagent I and Griess Reagent II, incubate at room temperature for 10 minutes and absorbance at 540 $\mathrm{nm}$ was measured. Nitrite concentrations were quantified by comparing the absorbance values to a standard curve generated by serial dilution of $100 \mathrm{uM}$ sodium nitrite.

\section{In vivo experiments}

C57BL/6 B16 melanoma mouse model was used to investigate the effect(s) of RNCR3-modified MDSCs on tumor growth. Mice were injected with $1 \times 10^{6} \mathrm{~B} 16$ and were randomly divided into several experimental groups (six mice/group), and then the prepared MDSCs $\left(1 \times 10^{6}\right)$ were injected into different groups via tail vein at day 5 , 
day 11 and day 18 after injection of tumor cells. For the preparation of RNCR3-modified MDSCs, BMCs obtained from C57BL/6 CD45.1 mice were first transfected with control siRNA or RNCR3 siRNA according to our previous method [44] or transduced by RNCR3 shRNA/ lentiviruses and then cultured with GM-CSF (40 ng/ml) plus IL-6 $(40 \mathrm{ng} / \mathrm{ml})$ for 4 days. The tumor volume were measured in two dimensions by calipers every 2 days and calculated by the following formula: Width ${ }^{2} \times$ Length $\times \pi / 6$.

\section{RNA extraction and $q$ RT-PCR}

Total RNA was extracted from cells by using TRIzol reagent (Life Technologies, Carlsbad, CA) and was transcribed to cDNA using HiFiScript cDNA Synthesis Kit (CWBIO, Beijing, China) according to the manufacturer's instructions. The quantitative real-time PCR (qRT-PCR) was performed by using Hieff ${ }^{\mathrm{TM}}$ qPCR SYBR-Green Master Mix (YEASEN, Shanghai, China) in a Bio-Rad iQ5 multicolour RT-PCR system. The primers used for qRT-PCR were showed in Supplementary Table 1. The levels of each gene was calculated using the $2^{-\Delta \Delta C T}$ method. The stem-loop RT primer (5'- GTCGTATCCAG TGCAGGGTCCGAGGTATTCGCACTGGATACGACTC AGGA-3') was used for miR-185-5p level detection. The relative expression level of miR-185-5p was normalized to that of internal control U6. For other genes, GAPDH was used as the endogenous control.

\section{Flow cytometric analyses}

Cells were collected and washed twice with PBS, and then incubated in PBS with 1\% FBS and antibodies for 30min. After washing twice with PBS, cells were fixed in $1 \%$ paraformaldehyde and analyzed by the FACScan flow cytometer (BD Biosciences). Anti-Gr1FITC(RB6-8C5), CD11b-FITC(M1/70), Ly6G-PE(1A8), Ly6C-FITC(AL-21) and CD45.1-APC(A20) were purchased from BD Biosciences (San Diego, CA). AntiCD11b-PercP/cy5.5(M1/70), Gr1-PE(RB6-8C5), CD45APC(30-F11), CD4-PE(GK1.5), CD8a-FITC(53-6.7) and 7 -AAD were purchased from BioLegend (San Diego, CA).

\section{Western blot}

Western blot was performed as described previously [45]. Hybridizations with primary Abs were carried out for $1 \mathrm{hr}$ at room temperature in blocking buffer. The protein-Ab complexes were detected using peroxidaseconjugated secondary Abs (Boehringer Mannheim) and ECL (Amersham Biosciences). Antibodies against Chop (Cell Signaling Technology, Boston, MA, USA, 1:2000 dilution), Arg-1(Santa Cruz Biotechnology, Santa Cruz, CA, 1:1000 dilution), iNOS (Santa Cruz Biotechnology, Santa Cruz, CA, 1:1000 dilution) and $\beta$-actin (Santa Cruz Biotechnology, Santa Cruz, CA, 1:1000 dilution) were used.

\section{RNA fluorescence in situ hybridization}

RNA fluorescence in situ hybridization (RNA-FISH) was performed according to reported protocol [46]. The cells first grew to desired confluency on sterile, $0.01 \%$ polylysine-treated glass slips in the bottom of a 6-well tissue culture dish. Aspirate the media and pipette $2 \mathrm{ml}$ of ice-cold CSK buffer into 6-well plate for 30s, $2 \mathrm{ml}$ of ice-cold CSK $+0.4 \%$ Triton X-100 buffer for 30s, $2 \mathrm{ml}$ of ice-cold CSK buffer for 30 s for cell membrane perforation. Pipette $2 \mathrm{ml}$ of $4 \%$ PFA for $10 \mathrm{~min}$, washed with cold $70 \%$ ethanol three times for cell fixation. The slides were dehydrated by moving them through a room temperature ethanol series $(85,95$, and $100 \%$ ethanol) for $2 \mathrm{~min}$ each, and air-dried at room temperature for $15 \mathrm{~min}$ and hybridized using the indicated probes overnight at $37^{\circ} \mathrm{C}$ in a humid chamber. After washing with $2 \times \mathrm{SSC} / 50 \%$ formamide, $2 \times \mathrm{SSC}$, and $1 \times$ SSC each for three times, DAPI dye was added. Seal the slides and observed using confocal microscope. The probe sequences were showed in Supplementary Table 1.

\section{Dual luciferase assay}

WT-3'UTR-Chop, WT-RNCR3, MUT(mutated)3'UTR-Chop and MUT-RNCR3 plasmids were constructed by cloning WT and mutated 3'UTR sequence of Chop or WT and mutated RNCR3 sequence into downstream of firefly luciferase cassette in $\mathrm{pSiCHECK}-2$ vector (Promega). The mutated sites of both Chop 3'UTR and RNCR3 were in miR-185-5p binding sites. The Chop 3'UTR and RNCR3 mutants were generated according to our previous method [47]. The primers used were listed in Supplementary Table 1. Mutations were confirmed by plasmid DNA sequencing. HEK293T cells were cultured in a 24 -well plate at $1 \times 10^{5}$ cells per well and then co-transfected with WT-3'UTRChop or MUT-3'UTR-Chop and miR-185-5p mimic or mimic control, or WT-RNCR3 or MUT-RNCR3 and miR185-5p mimic or mimic control with using Lipofectamine 2000 (Invitrogen). After transfection for 48h, relative luciferase activity was calculated by normalizing Firefly luminescence to Renilla luminescence using a DualLuciferase Reporter Assay (Promega, Madison, USA) according to the manufacturer's instructions.

\section{Statistical analyses}

All quantitative data were expressed as mean \pm SEM. A Student $t$ test and one-way ANOVA were used to determine statistical differences. A 95\% confidence interval was considered significant and was defined as $p<0.05$.

\section{Author contributions}

R.Y. designed the research and wrote the paper; W. S. designed the research and wrote the paper, and conducted in vivo and in vitro experiments and immunoassay. Z. T., H. Q and Y. G. were involved in vivo 
experiments and immunoassay. X. S. and Y. Z. offered assistances for the animal experiments. All authors read and approved the final manuscript.

\section{CONFLICTS OF INTEREST}

The authors declare no conflicts of interest.

\section{FUNDING}

This research was supported by NSFC grants 31470876, 91442111, 91629102 and ISF-NSFC program 31461143010; a Ministry of Science and Technology grant (863 program, 2008AA02Z129); and the National Key Scientific Program (2011CB964902); the Program for Changjiang Scholars and Innovative Research Team in University (No. IRT13023) and State Key Laboratory of Medicinal Chemical Biology.

\section{REFERENCES}

1. Bronte V, Brandau S, Chen SH, Colombo MP, Frey AB, Greten TF, Mandruzzato S, Murray PJ, Ochoa A, OstrandRosenberg S, Rodriguez PC, Sica A, Umansky V, et al. Recommendations for myeloid-derived suppressor cell nomenclature and characterization standards. Nat Commun. 2016; 7:12150.

2. Kumar V, Patel S, Tcyganov E, Gabrilovich DI. The nature of myeloid-derived suppressor cells in the tumor microenvironment. Trends Immunol. 2016; 37:208-220.

3. Marvel D, Gabrilovich DI. Myeloid-derived suppressor cells in the tumor microenvironment: expect the unexpected. J Clin Invest. 2015; 125:3356-3364.

4. Gabrilovich DI. Myeloid-derived suppressor cells. Cancer Immunol Res. 2017; 5:3-8.

5. Cheng P, Kumar V, Liu H, Youn JI, Fishman M, Sherman $\mathrm{S}$, Gabrilovich D. Effects of notch signaling on regulation of myeloid cell differentiation in cancer. Cancer Res. 2014; 74:141-152.

6. Nagaraj S, Gupta K, Pisarev V, Kinarsky L, Sherman S, Kang L, Herber DL, Schneck J, Gabrilovich DI. Altered recognition of antigen is a mechanism of $\mathrm{CD} 8+\mathrm{T}$ cell tolerance in cancer. Nat Med. 2007; 13:828-835.

7. Raber PL, Thevenot P, Sierra R, Wyczechowska D, Halle D, Ramirez ME, Ochoa AC, Fletcher M, Velasco C, Wilk A, Reiss K, Rodriguez PC. Subpopulations of myeloidderived suppressor cells impair $\mathrm{T}$ cell responses through independent nitric oxide-related pathways. Int $\mathrm{J}$ Cancer. 2014; 134:2853-2864.

8. Schmielau J, Finn OJ. Activated granulocytes and granulocyte-derived hydrogen peroxide are the underlying mechanism of suppression of t-cell function in advanced cancer patients. Cancer Res. 2001; 61:4756-4760.
9. Raber P, Ochoa AC, Rodriguez PC. Metabolism of L-arginine by myeloid-derived suppressor cells in cancer: mechanisms of $\mathrm{T}$ cell suppression and therapeutic perspectives. Immunol Invest. 2012; 41:614-634.

10. Srivastava MK, Sinha P, Clements VK, Rodriguez P, Ostrand-Rosenberg S. Myeloid-derived suppressor cells inhibit T-cell activation by depleting cystine and cysteine. Cancer Res. 2010; 70:68-77.

11. Yu J, Du W, Yan F, Wang Y, Li H, Cao S, Yu W, Shen C, Liu J, Ren X. Myeloid-derived suppressor cells suppress antitumor immune responses through IDO expression and correlate with lymph node metastasis in patients with breast cancer. J Immunol. 2013; 190:3783-3797.

12. Zhao Y, Wu T, Shao S, Shi B, Zhao Y. Phenotype, development, and biological function of myeloid-derived suppressor cells. Oncoimmunology. 2016; 5:e1004983.

13. Condamine T, Gabrilovich DI. Molecular mechanisms regulating myeloid-derived suppressor cell differentiation and function. Trends Immunol. 2011; 32:19-25.

14. Di Mitri D, Toso A, Alimonti A. Molecular pathways: targeting tumor-infiltrating myeloid-derived suppressor cells for cancer therapy. Clin Cancer Res. 2015; 21:3108-3112.

15. Khaled YS, Ammori BJ, Elkord E. Myeloid-derived suppressor cells in cancer: recent progress and prospects. Immunol Cell Biol. 2013; 91:493-502.

16. Kapanadze T, Gamrekelashvili J, Ma C, Chan C, Zhao F, Hewitt S, Zender L, Kapoor V, Felsher DW, Manns MP, Korangy F, Greten TF. Regulation of accumulation and function of myeloid derived suppressor cells in different murine models of hepatocellular carcinoma. J Hepatol. 2013; 59:1007-1013.

17. Kong YY, Fuchsberger M, Xiang SD, Apostolopoulos V, Plebanski M. Myeloid derived suppressor cells and their role in diseases. Curr Med Chem. 2013; 20:1437-1444.

18. Marigo I, Bosio E, Solito S, Mesa C, Fernandez A, Dolcetti L, Ugel S, Sonda N, Bicciato S, Falisi E, Calabrese F, Basso $\mathrm{G}$, Zanovello P, et al. Tumor-induced tolerance and immune suppression depend on the $\mathrm{C} / \mathrm{EBP}$ beta transcription factor. Immunity. 2010; 32:790-802.

19. Harding HP, Zhang Y, Zeng H, Novoa I, Lu PD, Calfon M, Sadri N, Yun C, Popko B, Paules R, Stojdl DF, Bell JC, Hettmann T, et al. An integrated stress response regulates amino acid metabolism and resistance to oxidative stress. Mol Cell. 2003; 11:619-633.

20. Rzymski T, Harris AL. The unfolded protein response and integrated stress response to anoxia. Clin Cancer Res. 2007; 13:2537-2540.

21. Zhang X, Lian Z, Padden C, Gerstein MB, Rozowsky J, Snyder M, Gingeras TR, Kapranov P, Weissman SM, Newburger PE. A myelopoiesis-associated regulatory intergenic noncoding RNA transcript within the human HOXA cluster. Blood. 2009; 113:2526-2534. 
22. Kotzin JJ, Spencer SP, McCright SJ, Kumar DB, Collet MA, Mowel WK, Elliott EN, Uyar A, Makiya MA, Dunagin MC, Harman CC, Virtue AT, Zhu S, et al. The long non-coding RNA Morrbid regulates Bim and short-lived myeloid cell lifespan. Nature. 2016; 537:239-243.

23. Wang P, Xue Y, Han Y, Lin L, Wu C, Xu S, Jiang Z, Xu J, Liu Q, Cao X. The STAT3-binding long noncoding RNA lnc-DC controls human dendritic cell differentiation. Science. 2014; 344:310-313.

24. Xin J, Li J, Feng Y, Wang L, Zhang Y, Yang R. Downregulation of long noncoding RNA HOTAIRM1 promotes monocyte/dendritic cell differentiation through competitively binding to endogenous miR-3960. Onco Targets Ther. 2017; 10:1307-1315.

25. Mercer TR, Qureshi IA, Gokhan S, Dinger ME, Li G, Mattick JS, Mehler MF. Long noncoding RNAs in neuronal-glial fate specification and oligodendrocyte lineage maturation. BMC Neurosci. 2010; 11:14.

26. Blackshaw S, Harpavat S, Trimarchi J, Cai L, Huang H, Kuo WP, Weber G, Lee K, Fraioli RE, Cho SH, Yung R, Asch E, Ohno-Machado L, et al. Genomic analysis of mouse retinal development. PLoS Biol. 2004; 2:E247.

27. Sanuki R, Onishi A, Koike C, Muramatsu R, Watanabe S, Muranishi Y, Irie S, Uneo S, Koyasu T, Matsui R, Chérasse Y, Urade Y, Watanabe D, et al. miR-124a is required for hippocampal axogenesis and retinal cone survival through Lhx2 suppression. Nat Neurosci. 2011; 14:1125-1134.

28. Shan K, Jiang Q, Wang XQ, Wang YN, Yang H, Yao MD, Liu C, Li XM, Yao J, Liu B, Zhang YY, J Y, Yan B. Role of long non-coding RNA-RNCR3 in atherosclerosis-related vascular dysfunction. Cell Death Dis. 2016; 7:e2248.

29. Cao X, Zhang W, Gu S, Yu Y, Tao Q, Ye T. Induction of antitumor immunity and treatment of preestablished tumor by interleukin-6-gene-transfected melanoma cells combined with low-dose interleukin-2. J Cancer Res Clin Oncol. 1995; 121:721-728.

30. Thevenot PT, Sierra RA, Raber PL, Al-Khami AA, Trillo-Tinoco J, Zarreii P, Ochoa AC, Cui Y, Del Valle L, Rodriguez PC. The stress-response sensor chop regulates the function and accumulation of myeloid-derived suppressor cells in tumors. Immunity. 2014; 41:389-401.

31. Hu G, Gong AY, Wang Y, Ma S, Chen X, Chen J, Su CJ, Shibata A, Strauss-Soukup JK, Drescher KM, Chen XM. LincRNA-Cox 2 promotes late inflammatory gene transcription in macrophages through modulating SWI/ SNF-mediated chromatin remodeling. J Immunol. 2016; 196:2799-2808.

32. Atianand MK, Caffrey DR, Fitzgerald KA. Immunobiology of long noncoding RNAs. Annu Rev Immunol. 2017; 35:177-198.

33. Fatica A, Bozzoni I. Long non-coding RNAs: new players in cell differentiation and development. Nat Rev Genet. 2014; 15:7-21.
34. Bartel DP. MicroRNAs: target recognition and regulatory functions. Cell. 2009; 136:215-233.

35. Rani N, Nowakowski TJ, Zhou H, Godshalk SE, Lisi V, Kriegstein AR, Kosik KS. A primate lncRNA mediates notch signaling during neuronal development by sequestering miRNA. Neuron. 2016; 90:1174-1188.

36. Cai H, Yao J, An Y, Chen X, Chen W, Wu D, Luo B, Yang Y, Jiang Y, Sun D, He X. LncRNA HOTAIR acts a competing endogenous RNA to control the expression of notch 3 via sponging miR-613 in pancreatic cancer. Oncotarget. 2017; 8:32905-32917. https://doi.org/10.18632/oncotarget.16462.

37. Sonda N, Simonato F, Peranzoni E, Cali B, Bortoluzzi S, Bisognin A, Wang E, Marincola FM, Naldini L, Gentner B, Trautwein C, Sackett SD, Zanovello P, et al. miR-142-3p prevents macrophage differentiation during cancer-induced myelopoiesis. Immunity. 2013; 38:1236-1249.

38. Mildner A, Schonheit J, Giladi A, David E, Lara-Astiaso D, Lorenzo-Vivas E, Paul F, Chappell-Maor L, Priller J, Leutz A, Amit I, Jung S. Genomic characterization of murine monocytes reveals $\mathrm{C} / \mathrm{EBPbeta}$ transcription factor dependence of Ly6C- cells. Immunity. 2017; 46:849-862. e847.

39. Kumar V, Cheng P, Condamine T, Mony S, Languino LR, McCaffrey JC, Hockstein N, Guarino M, Masters G, Penman E, Denstman F, Xu X, Altieri DC, et al. CD45 phosphatase inhibits STAT3 transcription factor activity in myeloid cells and promotes tumor-associated macrophage differentiation. Immunity. 2016; 44:303-315.

40. Kortylewski M, Kujawski M, Wang T, Wei S, Zhang S, Pilon-Thomas S, Niu G, Kay H, Mule J, Kerr WG, Jove $\mathrm{R}$, Pardoll D, Yu H. Inhibiting Stat3 signaling in the hematopoietic system elicits multicomponent antitumor immunity. Nat Med. 2005; 11:1314-1321.

41. Nefedova Y, Nagaraj S, Rosenbauer A, Muro-Cacho C, Sebti SM, Gabrilovich DI. Regulation of dendritic cell differentiation and antitumor immune response in cancer by pharmacologic-selective inhibition of the janus-activated kinase 2/signal transducers and activators of transcription 3 pathway. Cancer Res. 2005; 65:9525-9535.

42. Pei K, Zhu JJ, Wang CE, Xie QL, Guo JY. MicroRNA185-5p modulates chemosensitivity of human non-small cell lung cancer to cisplatin via targeting ABCC1. Eur Rev Med Pharmacol Sci. 2016; 20:4697-4704.

43. Corzo CA, Cotter MJ, Cheng P, Cheng F, Kusmartsev S, Sotomayor E, Padhya T, McCaffrey TV, McCaffrey JC, Gabrilovich DI. Mechanism regulating reactive oxygen species in tumor-induced myeloid-derived suppressor cells. J Immunol. 2009; 182:5693-5701.

44. Xin J, Zhang Z, Su X, Wang L, Zhang Y, Yang R. Epigenetic component p66a modulates myeloid-derived suppressor cells by modifying STAT3. J Immunol. 2017; 198:2712-2720. 
45. Zeng B, Li H, Liu Y, Zhang Z, Zhang Y, Yang R. Tumorinduced suppressor of cytokine signaling 3 inhibits toll-like receptor 3 signaling in dendritic cells via binding to tyrosine kinase 2. Cancer Res. 2008; 68:5397-5404.

46. Hinten M, Maclary E, Gayen S, Harris C, Kalantry S. Visualizing long noncoding RNAs on chromatin. Methods Mol Biol. 2016; 1402:147-164.
47. Zhang M, Liu Q, Mi S, Liang X, Zhang Z, Su X, Liu J, Chen Y, Wang M, Zhang Y, Guo F, Zhang Z, Yang R. Both miR-17-5p and miR-20a alleviate suppressive potential of myeloid-derived suppressor cells by modulating STAT3 expression. J Immunol. 2011; 186:4716-4724. 